Article

\title{
Determination of Genetic Variations of Toll-Like Receptor (TLR) 2, 4, and 6 with Next-Generation Sequencing in Native Cattle Breeds of Anatolia and Holstein Friesian ${ }^{\dagger}$
}

\author{
Nuket Bilgen ${ }^{1, *}$, Bengi Cinar Kul ${ }^{1}$, Victoria Offord ${ }^{2}$, Dirk Werling ${ }^{2}$ and Okan Ertugrul ${ }^{1}$ \\ 1 Department of Animal Genetics, Faculty of Veterinary Medicine, University of Ankara, \\ Ankara 06610, Turkey; bkul@ankara.edu.tr (B.C.K.); oertugrul@ankara.edu.tr (O.E.) \\ 2 Department of Pathology and Infectious Diseases, Royal Veterinary College, University of London, \\ London AL9 7TA, UK; vofford@rvc.ac.uk (V.O.); dwerling@rvc.ac.uk (D.W.) \\ * Correspondence: nbilgen@ankara.edu.tr; Tel.: +90-312-317-0315 (ext. 4327) \\ + Work was carried out in fulfillment of the requirements for a Doctoral thesis, The Graduate School of Health \\ Sciences of Ankara University, Ankara 06110, Turkey.
}

Academic Editor: Michael Wink

Received: 27 May 2016; Accepted: 31 October 2016; Published: 3 November 2016

\begin{abstract}
In recent years, the focus of disease resistance and susceptibility studies in cattle have been on determining patterns in the innate immune response of key proteins, such as Toll-like receptors (TLR). In the bovine genome, there are 10 TLR family members and, of these, TLR2, TLR4, and TLR6 are specialized in the recognition of bacterial ligands. Indigenous cattle breeds of Anatolia have been reported to show fewer signs of clinical bacterial infections, such as bovine tuberculosis and mastitis, and it is hypothesized that this might be due to a less stringent genetic selection during breeding. In contrast, Holstein-Friesian cattle have been under strong selection for milk production, which may have resulted in greater susceptibility to diseases. To test this hypothesis, we have compared the TLR2, TLR4, and TLR6 genes of Anatolian Black (AB), East Anatolian Red (EAR), South Anatolian Red (SAR), Turkish Grey (TG), and Holstein (HOL) cattle using next-generation sequencing. The SAR breed had the most variations overall, followed by EAR, AB, TG, and HOL. TG had the most variations for TLR2, whereas SAR had the most variations in TLR4 and TLR6. We compared these variants with those associated with disease and susceptibility traits. We used exon variants to construct haplotypes, investigated shared haplotypes within breeds, and proposed candidate haplotypes for a disease resistance phenotype in Anatolian cattle breeds.
\end{abstract}

Keywords: Anatolian Black; East Anatolian Red; South Anatolian Red; Turkish Grey; Holstein Friesian; innate immunity; next generation sequencing; TLR2; TLR4; TLR6

\section{Introduction}

Toll-like receptors (TLRs) recognize conserved patterns in diverse microbial molecules called microbial-associated molecular patterns (MAMPs). These include lipopolysaccharide recognized by TLR 4 and lipopeptides recognized by the heterodimer formed by TLR 2 with either TLR1 or TLR6 [1]. In addition, TLRs also react to damage-associated molecular patterns (DAMPs) [2,3] released after cellular damage and, thus, play a crucial role in initiating the innate immune response [4]. Genetic variations found in the genes encoding TLRs have been associated with disease susceptibility and resistance in a variety of animal species $[1,5,6]$. In the bovine genome, 10 members of the TLR family (TLR1-10) have been identified and mapped to specific chromosomes [7]. Bovine TLR2, TLR4, and TLR6 genes are located on BTA17, BTA8, and BTA6, respectively, and have defined amino acid 
(aa) lengths [8,9]. Independent of their aa length, all TLRs identified so far contain three domains: an extra-cellular ligand binding domain (ECD) consisting of various numbers of leucine-rich repeats (LRR), a trans-membrane domain (TMD), and an intra-cellular domain, which is also known as the Toll/Interleukin-1 Receptor (TIR) domain [10], of which the TMD and TIR domain seem to be highly conserved between species [11].

Several studies have suggested that disease resistance, susceptibility, and severity of clinical signs in individuals or breeds may be attributed to altered ligand binding caused by single nucleotide polymorphisms (SNPs) specifically within the ECD of TLR genes [11,12]. Given the increased use of antibiotics in food-producing animals, native cattle breeds could provide an important genetic resource to breeders. Many of these indigenous breeds show an enhanced disease resistance due to a co-evolution with specific pathogens over decades, potentially resulting in the development of genetic resistance. Indeed, due to low selective pressure, local breeds are generally not as productive as high-yielding breeds selected for specific QTLs and, thus, may have preserved their genetic makeup over the years.

Anatolia has five native cattle breeds; Anatolian Black (AB), South Anatolian Red (SAR), East Anatolian Red (EAR), Turkish Grey (TG), and Native Southern Yellow. In general, these breeds grow slowly, are adapted to extensive breeding conditions, and present the morphologically look of semi-wild cattle [13]. Milk production in these breeds is between 700-1000 kg per lactation period per cow. The breeds are adapted to challenging environmental conditions and are known to be resistant to specific diseases. For example, SAR and EAR breeds are known to be resistant to blood parasite infections [14], whereas cows from the AB breed showed an extremely low bovine tuberculosis incidence rate when kept with Mycobacterium bovis-infected cattle [15], as well as a low mastitis-incidence rate [15]. As the corresponding disease-causing bacteria have been described to bind to TLR2, TLR4, and TLR6, the aim of the study was to determine the presence of SNP in these genes in the $\mathrm{AB}, \mathrm{EAR}, \mathrm{SAR}$, and TG indigenous breeds compared to those present in the corresponding genes of HOL cattle.

\section{Materials and Methods}

\subsection{DNA Isolation and Amplicon Sequencing}

To determine variations in the genomic sequences for TLR2, TLR4, and TLR6, blood samples from $\mathrm{AB}(n=20)$, EAR $(n=20), \mathrm{SAR}(n=20), \mathrm{TG}(n=10)$, and HOL $(n=10)$ were used. Blood samples were collected from each breed across Turkey (Figure 1).

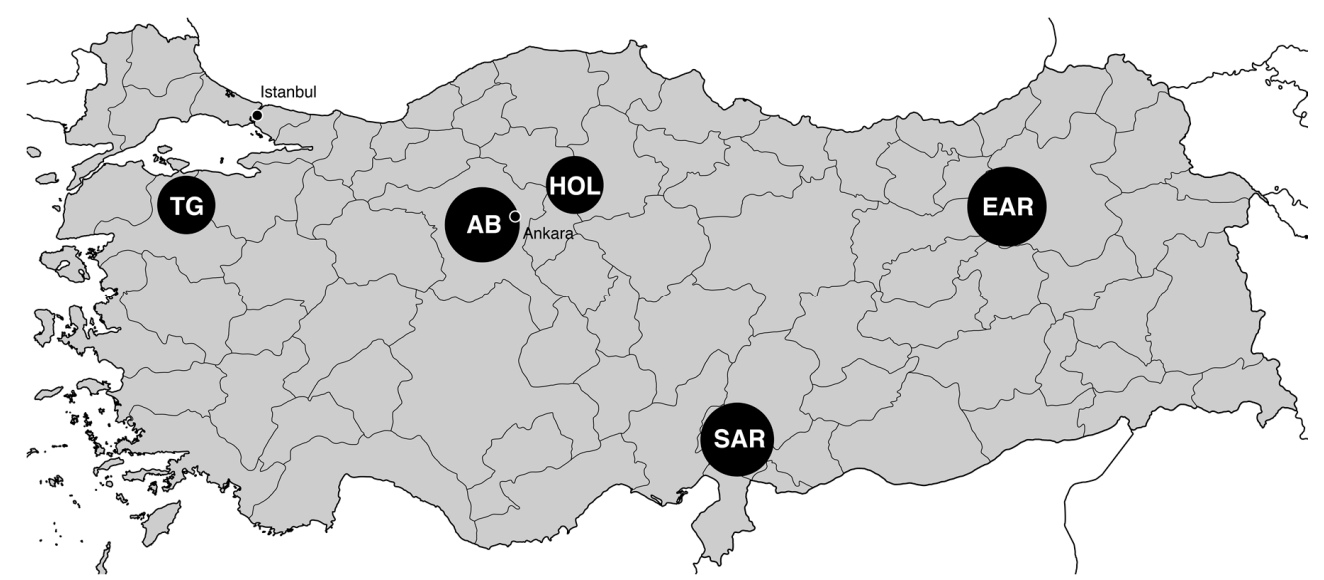

Figure 1. Geographical regions of the sampled breeds: Anatolian Black (AB), East Anatolian Red (EAR), South Anatolian Red (SAR), Turkish Grey (TG), and Holstein (HOL). Sizes of circles denote relative population sizes. 
Genomic DNA (gDNA) extraction was performed using a commercially available gDNA extraction kit (Qiagen Blood and Tissue, Hilden, Germany). Extracted DNA was measured by spectrophotometry (Nanodrop 2000, Thermo, Wilmington, DE, USA) and integrity was visualized by agarose gel electrophoresis. To obtain reliable variant information, paired end sequencing was performed at 50× coverage to analyze TLR2, TLR4, and TLR6, which spanned 13, 11, and $19 \mathrm{~kb}$ regions, respectively. Different primer pair combinations were used for each gene (for details see: Table S1). Primers were designed using the Primer 3 software package [16] and were spaced out over 2500-3500 bp intervals. Hot start taq DNA polymerase (Phire Hot Start II, Thermo Fisher, Bremen, Germany) was used in PCR applications. PCR conditions were as followed; $95{ }^{\circ} \mathrm{C} 60 \mathrm{~s}$, for 45 cycles; $95{ }^{\circ} \mathrm{C} 10 \mathrm{~s}, 60{ }^{\circ} \mathrm{C} 120 \mathrm{~s}, 72{ }^{\circ} \mathrm{C} 20 \mathrm{~s}$, and final elongation at $72{ }^{\circ} \mathrm{C} 60 \mathrm{~s}$. For GC-rich regions $5 \%$ DMSO was added to the reaction to enhance PCR results (for details see: Table S2). PCR amplicons were visualized using SybrSafe (Invitrogen, Paisley, Scotland, UK) stained agarose gels. NGS library preparation was performed following the manufacturer's instructions (Nextera Library Preparation Kit, Illumina Inc., San Diego, CA, USA). Amplicons were sequenced using paired end sequencing on the MiSeq platform (Illumina in Intergen Lab Inc., Ankara, Turkey), and reads were aligned to the present bovine genome version available Btau 4.6.1 (Btau7) using the MiSeq software (Illumina, San Diego, CA, USA). The resulting alignment was used to construct binary-aligned map (bam) files.

\subsection{Sequence Analysis, Variant Verification, and Protein Modelling}

TLR2, TLR4, and TLR6 sequence datasets from individuals were analyzed using Picard [17], BAM tools, SAM tools [18], and GATK [19] to generate variation call files (VCF) for each individual [20]. SnpSift [21] and SnpEff analysis tools [22] were used to annotate variants with the SNP138 variant collection. Thereafter, VCFs were aggregated at the breed level to determine novel SNP and InDel variants, and the results were stored in distinct .vcf files. Positions of both SNPs and InDels were lifted over to a newer assembly version (Btau8) in UCSC [8] and new positions were uploaded to the Ensembl Variant Effect Predictor (VEP) [23] database. The accuracy and the annotation of the identified genetic variations were assessed using VEP [23] and SnpSift [21], respectively.

Although VEP [23] and SnpSift [21] are useful tools to assess accuracy and to annotate novel candidate SNPs, we also screened variants using their coverage and frequency to eliminate false variant calls. Potentially important non-synonymous and novel SNP variants located in exons were validated subsequently by using Sanger sequencing. New sets of primers covering the regions including variants were designed by using Primer3 [16] (Table S1). The PCR products were sequenced using a BigDye Terminator v3.1 cycle sequencing kit and ABI310 automatic sequencer (Applied Biosystems, Foster City, CA, USA). Amplification primers were used for bidirectional sequencing. Obtained data was analyzed using BioEdit software [24].

After analyzing the variants at the amino acid level, a protein model was constructed for the most important variant identified in TLR2. Models were constructed using the Modeller [25] software package, and validated using ProCheck [26], Verify 3D [27], ERRAT [28], and ProQ [29].

\section{Results}

Obtained .bam files were visualized using Integrative Genomics Viewer (IGV) [30]. The average read length was determined to be $125 \mathrm{bp}$. Some .bam files contained ambiguous alignments due to the presence of nonspecific amplicons produced during the amplification of gene regions. Thus, all ambiguous alignments and reads with less than $125 \mathrm{bp}$ were removed and filtered according to their mapping quality before calling variants using $M Q>50$. Across all analyzed sequences from all breeds, five intronic regions in 13 individuals could not be amplified and, therefore, NGS results are missing for these individuals. Furthermore, one individual from the HOL breed appeared to have variations found only in Anatolian breeds and was subsequently excluded from the analysis.

Within the three TLR genes analyzed, a total of 360, 463, 520, 423, and 274 SNP variants were determined at the breed level in AB, EAR, SAR, TG, and HOL individuals used in this study, 
including 26, 23, 68, 22, and four novel SNPs (Table 1). According to average data on determined SNPs and genes, the highest SNP variation was found in the TLR6 gene, whereas the lowest one was found in TLR4 (Figure 2).

Table 1. SNP variants identified in TLR2, TLR4, and TLR6 genes. In each cell of the table, the total/novel number of SNPs is shown, or only the total if no novel SNPs were found.

\begin{tabular}{cccccc}
\hline \multirow{2}{*}{ Gene } & \multicolumn{5}{c}{ Breed } \\
\cline { 2 - 6 } & AB & EAR & SAR & TG & HOL \\
\hline TLR2 & $133 / 11$ & $153 / 17$ & $155 / 13$ & $187 / 16$ & $45 / 3$ \\
Exon & 23 & 27 & 28 & 26 & 6 \\
missense & 13 & 16 & $17 / 1$ & $15 / 1$ & 4 \\
synonymous & 10 & $11 / 1$ & $11 / 1$ & 11 & 2 \\
Intron & $93 / 10$ & $111 / 15$ & $110 / 10$ & $146 / 15$ & $34 / 3$ \\
3'UTR & $15 / 1$ & $13 / 1$ & $15 / 1$ & 14 & 4 \\
5'UTR & 2 & 2 & 2 & 1 & 1 \\
\hline TLR6 & $209 / 14$ & $230 / 5$ & $285 / 52$ & $179 / 5$ & 155 \\
Exon & 26 & 23 & 26 & 24 & 9 \\
missense & $10 / 2$ & $9 / 1$ & $9 / 1$ & $8 / 1$ & 3 \\
synonymous & $16 / 1$ & 14 & $17 / 2$ & $16 / 1$ & 6 \\
Intron & $177 / 8$ & $203 / 4$ & $252 / 46$ & $153 / 2$ & 103 \\
3'UTR & 2 & 1 & $3 / 2$ & 2 & 1 \\
5'UTR & $4 / 3$ & 4 & $5 / 1$ & $1 / 1$ & 2 \\
\hline TLR4 & $18 / 1$ & $80 / 1$ & $80 / 3$ & $57 / 1$ & $74 / 1$ \\
Exon & 2 & 16 & 13 & 10 & 15 \\
missense & 1 & 6 & 2 & $3 / 1$ & 5 \\
synonymous & 1 & 10 & 11 & 7 & 10 \\
Intron & 14 & $59 / 1$ & $62 / 3$ & 45 & $55 / 1$ \\
3'UTR & 1 & 2 & 2 & 1 & 1 \\
5'UTR & 1 & 3 & 5 & 1 & 5 \\
\hline
\end{tabular}

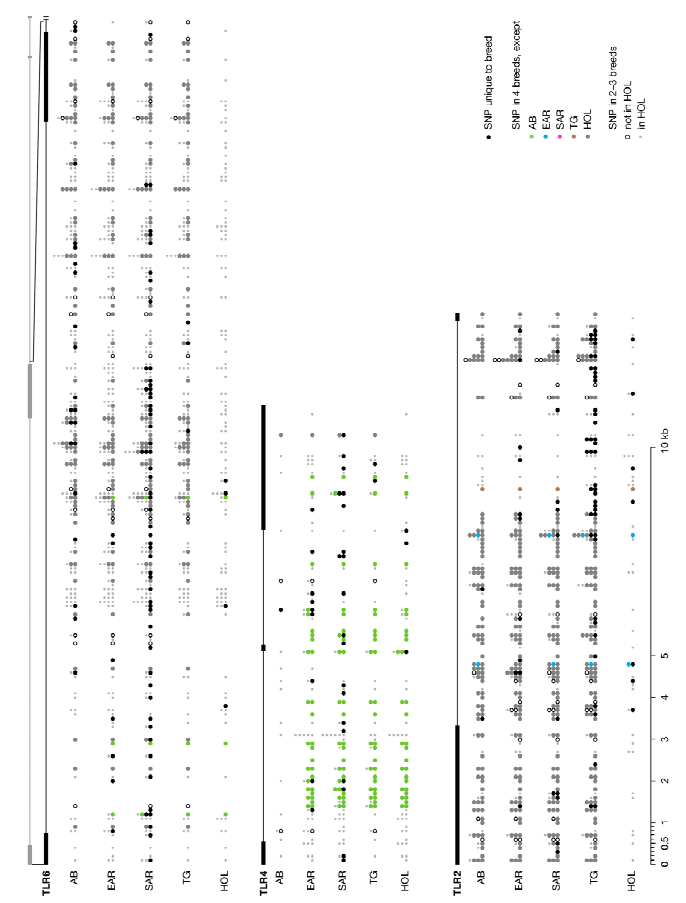

Figure 2. The location of SNPs in TLR6, TLR4 and TLR2 in five breeds of cattle: AB, EAR, SAR, TG, and HOL. SNPs are shown by circles formatted to emphasize their potential interest. In decreasing order of interest: SNPs unique to a breed (black), SNPs found in all breeds except one (colored by breed), SNPs found in 2-3 breeds and not in HOL (hollow), SNPs found in 2-3 breeds including HOL (small grey circle). Only the first two exons and first intron for TLR6 are shown. 
The SNP variants spread in the whole genes; however, InDel variants were identified only in intronic regions, suggesting no effect on the gene function (Table 2). Novel variants and variants potentially impacting on molecule structures identified by NGS results were subsequently confirmed using bidirectional Sanger sequencing (Figure 3).

Table 2. InDel variants identified in TLR2, TLR4, and TLR6 genes. In each cell of the table, the total/novel number of variants is shown.

\begin{tabular}{cccccc}
\hline \multirow{2}{*}{ Gene } & \multicolumn{5}{c}{ Breed } \\
\cline { 2 - 6 } & AB & EAR & SAR & TG & HOL \\
\hline TLR2 & $12 / 12$ & $16 / 13$ & $15 / 12$ & $16 / 13$ & $6 / 6$ \\
TLR6 & $23 / 9$ & $28 / 14$ & $38 / 24$ & $21 / 13$ & $17 / 3$ \\
TLR4 & $3 / 1$ & $5 / 3$ & $8 / 3$ & $6 / 4$ & $5 / 1$ \\
\hline
\end{tabular}
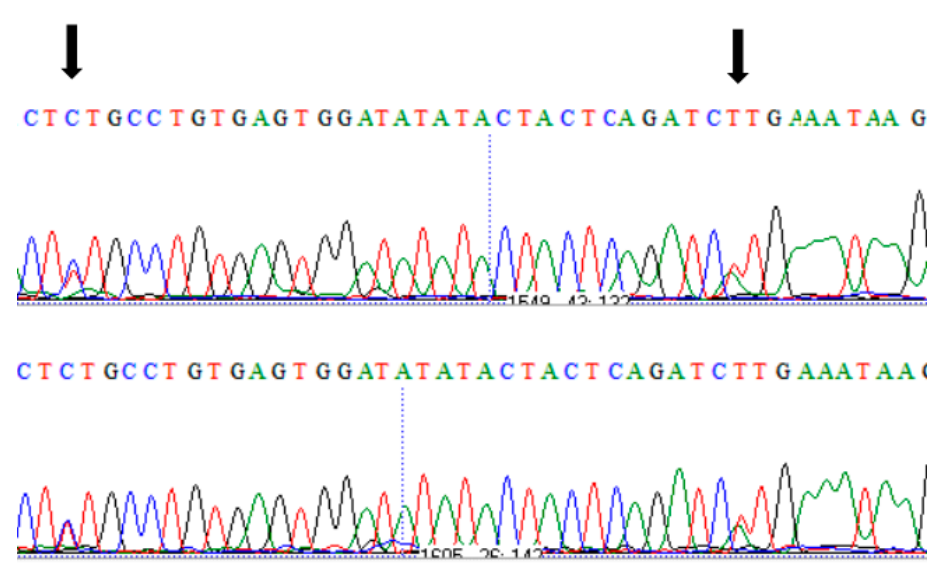

Figure 3. An example of the electropherogram, arrows showing R337Q and H326Q, respectively, in the AB breed.

By just analyzing SNPs occurring in the exons of TLR2, TLR4, and TLR6, a total of 33, 24, and 46 SNP variants covering the privates and the shared among breeds, respectively, were determined and according to these SNPs 36, 25, and 98 different haplotypes per corresponding TLR were constructed using ShapeIT software [31]. Obtained phased haplotypes (Table S3) were visualized in a median joining (MJ) tree using the Network program [32] (Figures 4 and 5), except TLR6, due to a high number of haplotypes. Within all haplotypes, two main haplotypes were identified for TLR2 and TLR6, whereas three major haplotypes were identified for TLR4. Analyzing haplotypes by breed, we identified 36 haplotypes in TLR2 for Anatolian breeds, but only four within HOL. Interestingly however, we were unable to identify any breed specific haplotypes for TLR4 and TLR6, but we were able to identify eight and 12 haplotypes, respectively, that were shared between Anatolian breeds and HOL (Table S3). 


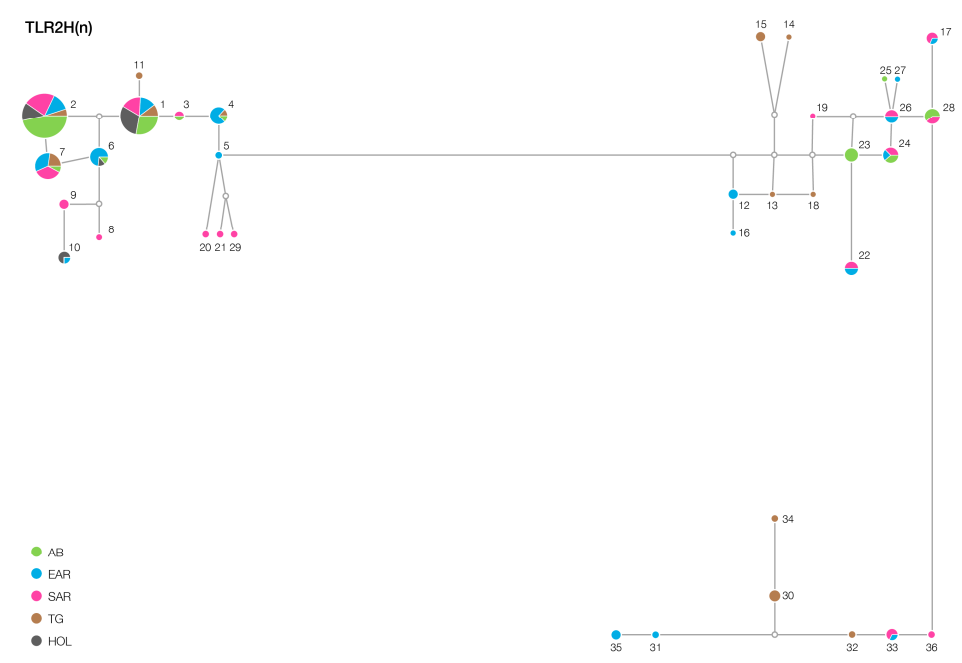

Figure 4. Median joining network constructed on the basis of 36 haplotype of TLR2. Filled circles represent haplotypes, and areas within circles are proportional to the number of individuals. The length of the lines connecting haplotypes and branching points correlates approximately with the number of base substitutions.

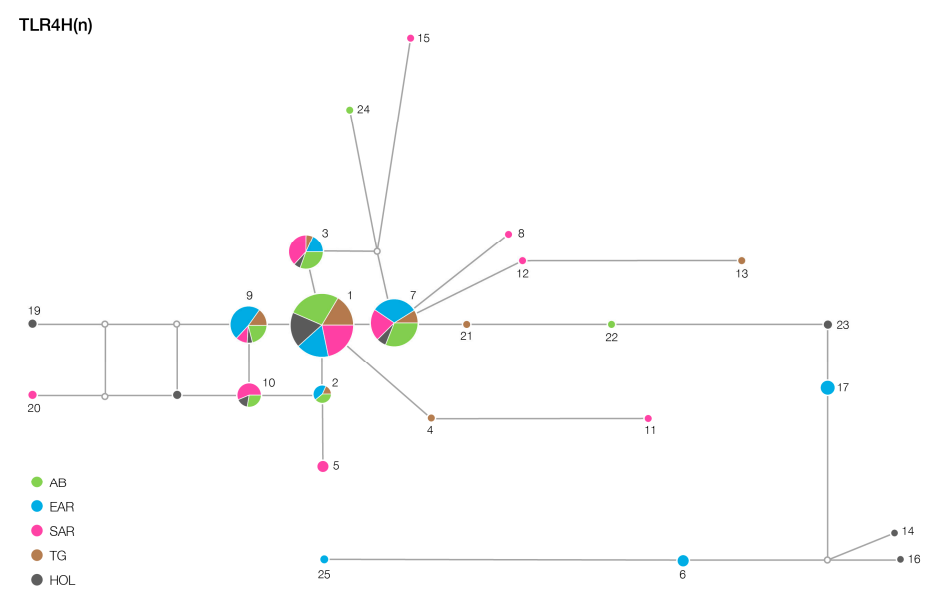

Figure 5. Median joining network constructed on the basis 25 haplotype of TLR4. Filled circles represent haplotypes, and areas within circles are proportional to the number of individuals. The length of the lines connecting haplotypes and branching points correlates approximately with the number of base substitutions.

Missense and synonymous variations were analyzed according to reference proteins for all genes and several changes were found in the analyzed genes affecting the amino acids characteristics (Tables 3-5).

Table 3. Missense and synonymous variations on protein domain level of TLR2 (according to reference sequence NP_776622.1).

\begin{tabular}{cccccccc}
\hline Domain & TLR2 (aa) & AB & EAR & SAR & TG & HOL & SNP ID \\
\hline LRR1 & $54 . .77$ & $62 \mathrm{~N} / \mathrm{N}$ & $62 \mathrm{~N} / \mathrm{N}$ & $62 \mathrm{~N} / \mathrm{N}$ & $62 \mathrm{~N} / \mathrm{N}$ & & rs68268249 \\
& & $63 \mathrm{E} / \mathrm{D}$ & $63 \mathrm{E} / \mathrm{D}$ & $63 \mathrm{E} / \mathrm{D}$ & $63 \mathrm{E} / \mathrm{D}$ & $63 \mathrm{E} / \mathrm{D}$ & rs55617172 \\
& & $68 \mathrm{G} / \mathrm{S}$ & $68 \mathrm{G} / \mathrm{S}$ & $68 \mathrm{G} / \mathrm{S}$ & $68 \mathrm{G} / \mathrm{S}$ & & rs68268250 \\
LRR3 & $102 . .125$ & & $119 \mathrm{~W} / \mathrm{L}$ & $119 \mathrm{~W} / \mathrm{L}$ & $119 \mathrm{~W} / \mathrm{L}$ & & rs211243949 \\
LRR5 & $151 . .175$ & & $152 \mathrm{R} / \mathrm{Q}$ & $152 \mathrm{R} / \mathrm{Q}$ & & $152 \mathrm{R} / \mathrm{Q}$ & rss3706434 \\
LRR7 & $200 . .223$ & & $201 \mathrm{~S} / \mathrm{N}$ & & & $201 \mathrm{~S} / \mathrm{N}$ & rs 110491977 \\
\hline
\end{tabular}


Table 3. Cont.

\begin{tabular}{|c|c|c|c|c|c|c|c|}
\hline Domain & TLR2 (aa) & $\overline{A B}$ & EAR & SAR & TG & HOL & SNP ID \\
\hline & & $211 \mathrm{I} / \mathrm{V}$ & $211 \mathrm{I} / \mathrm{V}$ & $211 \mathrm{I} / \mathrm{V}$ & $211 \mathrm{I} / \mathrm{V}$ & $211 \mathrm{I} / \mathrm{V}$ & rs43706433 \\
\hline LRR8 & $224 . .250$ & $227 \mathrm{~F} / \mathrm{L}$ & $227 \mathrm{~F} / \mathrm{L}$ & $227 \mathrm{~F} / \mathrm{L}$ & $227 \mathrm{~F} / \mathrm{L}$ & & rs68268251 \\
\hline LRR9-10 & $251 . .308$ & & & & & & \\
\hline \multirow[t]{2}{*}{ LRR11 } & $309 . .337$ & & & & $315 \mathrm{R} / \mathrm{R}$ & & rs68268253 \\
\hline & & $326 \mathrm{H} / \mathrm{Q}$ & $326 \mathrm{H} / \mathrm{Q}$ & $326 \mathrm{H} / \mathrm{Q}$ & $326 \mathrm{H} / \mathrm{Q}$ & & rs68343167 \\
\hline LRR12 & $338 . .361$ & $337 \mathrm{R} / \mathrm{Q}$ & $337 \mathrm{R} / \mathrm{Q}$ & $337 \mathrm{R} / \mathrm{Q}$ & $337 \mathrm{R} / \mathrm{Q}$ & & rs68343168 \\
\hline LRR13 & $362 . .388$ & & & & & & \\
\hline LRR14 & $389 . .414$ & $405 \mathrm{~T} / \mathrm{M}$ & $405 \mathrm{~T} / \mathrm{M}$ & $405 \mathrm{~T} / \mathrm{M}$ & $405 \mathrm{~T} / \mathrm{M}$ & & rs68268255 \\
\hline \multirow[t]{2}{*}{ LRR15 } & $415 . .437$ & $417 \mathrm{~N} / \mathrm{S}$ & 417 N/S & 417 N/S & 417 N/S & & rs68268256 \\
\hline & & $436 \mathrm{G} / \mathrm{G}$ & $436 \mathrm{G} / \mathrm{G}$ & $436 \mathrm{G} / \mathrm{G}$ & $436 \mathrm{G} / \mathrm{G}$ & & rs68268257 \\
\hline LRR16-18 & $438 . .500$ & & & & & & \\
\hline \multirow[t]{2}{*}{ LRR19 } & $501 . .524$ & $502 \mathrm{~S} / \mathrm{A}$ & $502 \mathrm{~S} / \mathrm{A}$ & $\begin{array}{l}502 \mathrm{~S} / \mathrm{A} \\
530 \mathrm{~A} / \mathrm{A}\end{array}$ & $502 \mathrm{~S} / \mathrm{A}$ & & $\begin{array}{c}\text { rs68268258 } \\
\text { novel }\end{array}$ \\
\hline & $533 . .586$ & $544 \mathrm{~F} / \mathrm{F}$ & $545 \mathrm{~F} / \mathrm{F}$ & $\begin{array}{l}531 \mathrm{G} / \mathrm{S} \\
546 \mathrm{~F} / \mathrm{F}\end{array}$ & $547 \mathrm{~F} / \mathrm{F}$ & $544 \mathrm{~F} / \mathrm{F}$ & $\begin{array}{c}\text { novel } \\
\text { rs68268259 }\end{array}$ \\
\hline \multirow{2}{*}{ LRR20 } & & $563 \mathrm{R} / \mathrm{H}$ & $563 \mathrm{R} / \mathrm{H}$ & $563 \mathrm{R} / \mathrm{H}$ & $563 \mathrm{R} / \mathrm{H}$ & $34+1 / 1$ & rs68268260 \\
\hline & $588 . .608$ & $569 \mathrm{H} / \mathrm{H}$ & $569 \mathrm{H} / \mathrm{H}$ & $\begin{array}{l}569 \mathrm{H} / \mathrm{H} \\
574 \mathrm{R} / \mathrm{W}\end{array}$ & $569 \mathrm{H} / \mathrm{H}$ & $569 \mathrm{H} / \mathrm{H}$ & $\begin{array}{c}\text { rs41830058 } \\
\text { novel }\end{array}$ \\
\hline \multirow{4}{*}{ Trans Membrane } & & $593 \mathrm{~A} / \mathrm{A}$ & $593 \mathrm{~A} / \mathrm{A}$ & $593 \mathrm{~A} / \mathrm{A}$ & $593 \mathrm{~A} / \mathrm{A}$ & & rs68268261 \\
\hline & & $594 \mathrm{~A} / \mathrm{A}$ & $594 \mathrm{~A} / \mathrm{A}$ & $594 \mathrm{~A} / \mathrm{A}$ & $594 \mathrm{~A} / \mathrm{A}$ & & rs68343169 \\
\hline & & $605 \mathrm{~T} / \mathrm{M}$ & $605 \mathrm{~T} / \mathrm{M}$ & $605 \mathrm{~T} / \mathrm{M}$ & $605 \mathrm{~T} / \mathrm{M}$ & & rs 68343170 \\
\hline & & & & & $634 \mathrm{~A} / \mathrm{V}$ & & novel \\
\hline \multirow[t]{6}{*}{ TIR } & $640 . .784$ & & $644 \mathrm{~F} / \mathrm{F}$ & & $250 \mathrm{H}$ & & novel \\
\hline & & $665 \mathrm{H} / \mathrm{Q}$ & $665 \mathrm{H} / \mathrm{Q}$ & $665 \mathrm{H} / \mathrm{Q}$ & $665 \mathrm{H} / \mathrm{Q}$ & & rs68268263 \\
\hline & & $675 \mathrm{H} / \mathrm{H}$ & $675 \mathrm{H} / \mathrm{H}$ & $675 \mathrm{H} / \mathrm{H}$ & $675 \mathrm{H} / \mathrm{H}$ & & rs68343171 \\
\hline & & $685 \mathrm{I} / \mathrm{I}$ & $685 \mathrm{I} / \mathrm{I}$ & $685 \mathrm{I} / \mathrm{I}$ & $685 \mathrm{I} / \mathrm{I}$ & & rs68268264 \\
\hline & & $738 \mathrm{E} / \mathrm{Q}$ & $738 \mathrm{E} / \mathrm{Q}$ & $738 \mathrm{E} / \mathrm{Q}$ & & & rs207552166 \\
\hline & & $738 \mathrm{E} / \mathrm{E}$ & $738 \mathrm{E} / \mathrm{E}$ & $738 \mathrm{E} / \mathrm{E}$ & $738 \mathrm{E} / \mathrm{E}$ & & rs68268266 \\
\hline ATG16Lmotif & $761 . .778$ & $765 \mathrm{P} / \mathrm{P}$ & $765 \mathrm{P} / \mathrm{P}$ & $765 \mathrm{P} / \mathrm{P}$ & $765 \mathrm{P} / \mathrm{P}$ & & rs68268267 \\
\hline
\end{tabular}

Table 4. Missense and synonymous variations on protein domain level of TLR4 (according to reference sequence NP_776623.5).

\begin{tabular}{|c|c|c|c|c|c|c|c|}
\hline Domain & TLR4 (аa) & AB & EAR & SAR & TG & HOL & SNP ID \\
\hline LRR1 & $55 . .76$ & & & & & & \\
\hline LRR2 & $79 . .100$ & & & & & & \\
\hline LRR3 & 103..124 & & & & & & \\
\hline LRR4 & $127 . .148$ & & & & & & \\
\hline LRR5 & $151 . .172$ & & $151 \mathrm{~N} / \mathrm{T}$ & & & $151 \mathrm{~N} / \mathrm{T}$ & rs8193049 \\
\hline LRR6 & $176 . .197$ & & & & & & \\
\hline \multirow[t]{4}{*}{ LRR7 } & $205 . .225$ & & & & & & \\
\hline & & & $238 \mathrm{~N} / \mathrm{K}$ & & & & rs8193050 \\
\hline & & & $276 \mathrm{~F} / \mathrm{F}$ & & & & rs8193051 \\
\hline & & & $347 \mathrm{~A} / \mathrm{E}$ & & & $347 \mathrm{~A} / \mathrm{E}$ & rs8193053 \\
\hline LRR8 & $352 . .373$ & & & & & & \\
\hline \multirow[t]{4}{*}{ LRR9 } & $374 . .394$ & & 374 P/P & $374 \mathrm{P} / \mathrm{P}$ & 374 P/P & $374 \mathrm{P} / \mathrm{P}$ & rs8193054 \\
\hline & & & & $381 \mathrm{~K} / \mathrm{R}$ & & & rs8193055 \\
\hline & & & & $385 \mathrm{~L} / \mathrm{L}$ & & & rs8193056 \\
\hline & & & & $389 \mathrm{G} / \mathrm{G}$ & $389 \mathrm{G} / \mathrm{G}$ & & rs8193057 \\
\hline LRR10 & $400 . .422$ & & & & & & \\
\hline LRR11 & $423 . .444$ & & & & & & \\
\hline LRR12 & $448 . .469$ & & & & & & \\
\hline LRR13 & $472 . .495$ & & & & $482 \mathrm{~S} / \mathrm{Y}$ & & novel \\
\hline LRR14 & $497 . .518$ & & $507 \mathrm{Q} / \mathrm{Q}$ & $507 \mathrm{Q} / \mathrm{Q}$ & $507 \mathrm{Q} / \mathrm{Q}$ & $507 \mathrm{Q} / \mathrm{Q}$ & rs8193059 \\
\hline LRR15 & $521 . .542$ & & & & & & \\
\hline LRR16 & $545 . .568$ & $552 \mathrm{~S} / \mathrm{S}$ & $552 \mathrm{~S} / \mathrm{S}$ & $552 \mathrm{~S} / \mathrm{S}$ & $552 \mathrm{~S} / \mathrm{S}$ & $552 \mathrm{~S} / \mathrm{S}$ & rs8193060 \\
\hline LRR17 & - & & & & & & \\
\hline LRR18 & - & & & & & & \\
\hline LRR19 & - & & & & & & \\
\hline LRRCT & $579 . .626$ & & & $589 \mathrm{~S} / \mathrm{S}$ & & & rs8193061 \\
\hline
\end{tabular}


Table 4. Cont.

\begin{tabular}{|c|c|c|c|c|c|c|c|}
\hline Domain & TLR4 (aa) & $\mathbf{A B}$ & EAR & SAR & TG & HOL & SNP ID \\
\hline \multirow{9}{*}{ Trans membrane } & & & $609 \mathrm{C} / \mathrm{C}$ & & & $609 \mathrm{C} / \mathrm{C}$ & rs8193062 \\
\hline & & & & & $622 \mathrm{~S} / \mathrm{S}$ & & rs8193063 \\
\hline & & & $625 \mathrm{~N} / \mathrm{N}$ & & & $625 \mathrm{~N} / \mathrm{N}$ & rs8193064 \\
\hline & & & $649 \mathrm{G} / \mathrm{G}$ & & $649 \mathrm{G} / \mathrm{G}$ & 649 G/G & rs8193065 \\
\hline & & & $640 \mathrm{~V} / \mathrm{I}$ & & & $640 \mathrm{~V} / \mathrm{I}$ & rs8193066 \\
\hline & $633 . .653$ & & & & & & \\
\hline & & & $664 \mathrm{G} / \mathrm{G}$ & & & $664 \mathrm{G} / \mathrm{G}$ & rs8193067 \\
\hline & & $674 \mathrm{~T} / \mathrm{I}$ & $674 \mathrm{~T} / \mathrm{I}$ & $674 \mathrm{~T} / \mathrm{I}$ & $674 \mathrm{~T} / \mathrm{I}$ & $674 \mathrm{~T} / \mathrm{I}$ & rs8193069 \\
\hline & & & & 676 D/D & & & rs 8193070 \\
\hline
\end{tabular}

Table 5. Missense and synonymous variations on protein domain level of TLR6 (according to reference sequence NP_001001159.1).

\begin{tabular}{|c|c|c|c|c|c|c|c|}
\hline Domain & TLR6 (aa) & AB & EAR & SAR & TG & HOL & SNP ID \\
\hline LRR_RI & $<43 . .164$ & $37 \mathrm{D} / \mathrm{N}$ & & $37 \mathrm{D} / \mathrm{N}$ & $37 \mathrm{D} / \mathrm{N}$ & & \\
\hline LRR1 & $54 . .77$ & $61 \mathrm{Q} / \mathrm{Q}$ & $61 \mathrm{Q} / \mathrm{Q}$ & $61 \mathrm{Q} / \mathrm{Q}$ & $61 \mathrm{Q} / \mathrm{Q}$ & & rs68268271 \\
\hline LRR2 & 78..101 & $87 \mathrm{R} / \mathrm{G}$ & $87 \mathrm{R} / \mathrm{G}$ & 87 R/G & 87 R/G & & rs68268272 \\
\hline LRR3 & $102 . .122$ & $116 \mathrm{~S} / \mathrm{P}$ & $116 \mathrm{~S} / \mathrm{P}$ & & & & \\
\hline LRR4 & $123 . .147$ & $135 \mathrm{D} / \mathrm{H}$ & $135 \mathrm{D} / \mathrm{H}$ & $135 \mathrm{D} / \mathrm{H}$ & $135 \mathrm{D} / \mathrm{H}$ & & rs520121582 \\
\hline LRR5-LRR6 & $148 . .196$ & & & & & & \\
\hline LRR7 & $197 . .219$ & $\begin{array}{l}214 \mathrm{D} / \mathrm{N} \\
217 \mathrm{~A} / \mathrm{A}\end{array}$ & $\begin{array}{l}214 \mathrm{D} / \mathrm{N} \\
217 \mathrm{~A} / \mathrm{A}\end{array}$ & $\begin{array}{l}214 \mathrm{D} / \mathrm{N} \\
217 \mathrm{~A} / \mathrm{A}\end{array}$ & $\begin{array}{l}214 \mathrm{D} / \mathrm{N} \\
217 \mathrm{~A} / \mathrm{A}\end{array}$ & $214 \mathrm{D} / \mathrm{N}$ & $\begin{array}{l}\text { rs43702941 } \\
\text { rs68268273 }\end{array}$ \\
\hline LRR8-LRR12 & 220.354 & & & & & & \\
\hline LRR13 & $355 . .378$ & 374 D/D & & & $374 \mathrm{D} / \mathrm{D}$ & $374 \mathrm{D} / \mathrm{D}$ & rs68268274 \\
\hline \multirow[t]{2}{*}{ LRR14 } & $379 . .404$ & $395 \mathrm{~T} / \mathrm{A}$ & $395 \mathrm{~T} / \mathrm{A}$ & $395 \mathrm{~T} / \mathrm{A}$ & $395 \mathrm{~T} / \mathrm{A}$ & & rs68268275 \\
\hline & & $400 \mathrm{~K} / \mathrm{K}$ & $400 \mathrm{~K} / \mathrm{K}$ & $400 \mathrm{~K} / \mathrm{K}$ & $400 \mathrm{~K} / \mathrm{K}$ & & rs211657505 \\
\hline LRR15 & $405 . .428$ & $425 \mathrm{~S} / \mathrm{S}$ & $425 \mathrm{~S} / \mathrm{S}$ & $425 \mathrm{~S} / \mathrm{S}$ & $425 \mathrm{~S} / \mathrm{S}$ & & rs68268276 \\
\hline LRR16 & $429 . .449$ & & & & & & \\
\hline LRR17 & $450 . .473$ & & $458 \mathrm{H} / \mathrm{H}$ & $458 \mathrm{H} / \mathrm{H}$ & & & rs68268277 \\
\hline LRR18 & $474 . .495$ & & & & & & \\
\hline \multirow[t]{3}{*}{ LRR19 } & $496 . .519$ & $505 \mathrm{~N} / \mathrm{N}$ & $505 \mathrm{~N} / \mathrm{N}$ & $505 \mathrm{~N} / \mathrm{N}$ & $505 \mathrm{~N} / \mathrm{N}$ & & rs55617146 \\
\hline & & $526 \mathrm{~V} / \mathrm{A}$ & $526 \mathrm{~V} / \mathrm{A}$ & $526 \mathrm{~V} / \mathrm{A}$ & $526 \mathrm{~V} / \mathrm{A}$ & $526 \mathrm{~V} / \mathrm{A}$ & rs68343174, rs133754378 \\
\hline & & $526 \mathrm{~V}$ & $526 \mathrm{~V}$ & $526 \mathrm{~V}$ & $526 \mathrm{~V}$ & $526 \mathrm{~V}$ & rs68343175, rs136574510 \\
\hline \multirow[t]{3}{*}{ LRRCT } & $529 . .582$ & $539 \mathrm{D} / \mathrm{D}$ & $539 \mathrm{D} / \mathrm{D}$ & $539 \mathrm{D} / \mathrm{D}$ & & & rs68343176 \\
\hline & & & $544 \mathrm{~V} / \mathrm{I}$ & $544 \mathrm{~V} / \mathrm{I}$ & & & rs55617465, rs68268279 \\
\hline & & $573 \mathrm{~K} / \mathrm{K}$ & $573 \mathrm{~K} / \mathrm{K}$ & $573 \mathrm{~K} / \mathrm{K}$ & $573 \mathrm{~K} / \mathrm{K}$ & & rs55617193 \\
\hline \multirow[t]{2}{*}{ Trans membrane } & $585 . .605$ & $589 \mathrm{~V} / \mathrm{I}$ & $589 \mathrm{~V} / \mathrm{I}$ & $589 \mathrm{~V} / \mathrm{I}$ & $589 \mathrm{~V} / \mathrm{I}$ & $589 \mathrm{~V} / \mathrm{I}$ & rs55617317, rs207882984 \\
\hline & & $605 \mathrm{~L} / \mathrm{L}$ & $605 \mathrm{~L} / \mathrm{L}$ & $605 \mathrm{~L} / \mathrm{L}$ & $605 \mathrm{~L} / \mathrm{L}$ & $605 \mathrm{~L} / \mathrm{L}$ & rs68268280, rs378853146 \\
\hline \multirow[t]{10}{*}{ TIR } & $641 . .784$ & $642 \mathrm{~F} / \mathrm{F}$ & $642 \mathrm{~F} / \mathrm{F}$ & $642 \mathrm{~F} / \mathrm{F}$ & $642 \mathrm{~F} / \mathrm{F}$ & & rs438448894 \\
\hline & & $669 \mathrm{I} / \mathrm{V}$ & $669 \mathrm{I} / \mathrm{V}$ & $669 \mathrm{I} / \mathrm{V}$ & $669 \mathrm{I} / \mathrm{V}$ & & rs210580164 \\
\hline & & $674 \mathrm{H} / \mathrm{H}$ & $674 \mathrm{H} / \mathrm{H}$ & $674 \mathrm{H} / \mathrm{H}$ & $674 \mathrm{H} / \mathrm{H}$ & $674 \mathrm{H} / \mathrm{H}$ & rs209572763 \\
\hline & & $676 \mathrm{R} / \mathrm{R}$ & $676 \mathrm{R} / \mathrm{R}$ & $676 \mathrm{R} / \mathrm{R}$ & $676 \mathrm{R} / \mathrm{R}$ & & rs68343178 \\
\hline & & $680 \mathrm{~A} / \mathrm{A}$ & & $680 \mathrm{~A} / \mathrm{A}$ & $680 \mathrm{~A} / \mathrm{A}$ & & novel \\
\hline & & $684 \mathrm{I} / \mathrm{I}$ & $684 \mathrm{I} / \mathrm{I}$ & $684 \mathrm{I} / \mathrm{I}$ & 684 I/I & & rs68343179 \\
\hline & & $700 \mathrm{~F} / \mathrm{F}$ & $700 \mathrm{~F} / \mathrm{F}$ & $700 \mathrm{~F} / \mathrm{F}$ & $700 \mathrm{~F} / \mathrm{F}$ & & rs55617339, rs211454671 \\
\hline & & $701 \mathrm{~V} / \mathrm{V}$ & $701 \mathrm{~V} / \mathrm{V}$ & $701 \mathrm{~V} / \mathrm{V}$ & $701 \mathrm{~V} / \mathrm{V}$ & $701 \mathrm{~V} / \mathrm{V}$ & rs207586910 \\
\hline & & & $709 \mathrm{~S} / \mathrm{S}$ & $709 \mathrm{~S} / \mathrm{S}$ & & & rs55617335 \\
\hline & & & & E/E 710 & & & rs68268282 \\
\hline
\end{tabular}

Impact of Identified SNPs on Protein Model

Within the identified SNPs, we identified in LRR11 of TLR2 an amino acid change, which resulted in the change from a charged residue into an uncharged residue. This area is part of the TLR2 ligand binding domain, spanning LRRS 9-12. We visualized the importance of this change by constructing a hybrid protein model for one $\mathrm{AB}$ individual, carrying the $\mathrm{H} 326 \mathrm{Q}$ change, and other variants with one HOL individual. The resulting model was visualized in PyMol [33] (Figure 6). 


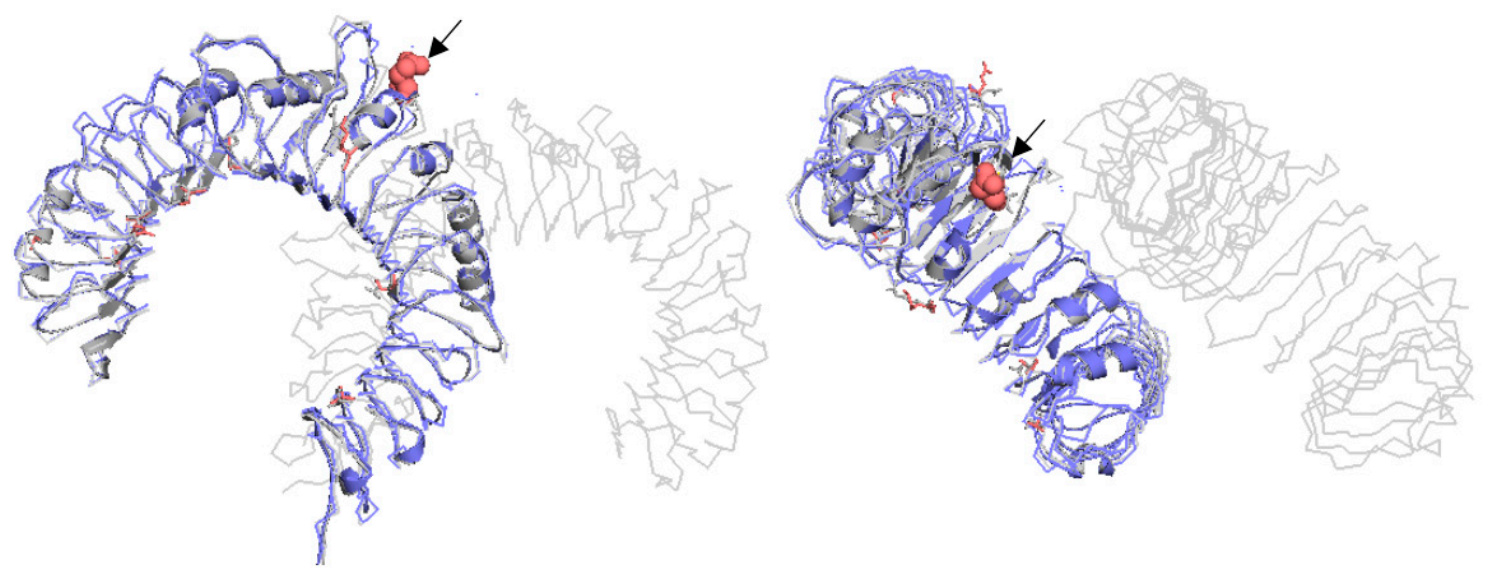

Figure 6. Predicted three-dimensional structures of homodimer of TLR2 representing the H326Q (arrows) variant. Blue: AB breed H326Q carrying individual, dark grey: one individual of HOL breed.

\section{Discussion}

The goal of the present study was to assess whether genetic variations in the sequences of innate immune receptors of indigenous cattle breeds compared to those sequences seen for these genes in HF cattle may potentially explain some of the observed genetic resistance to specific bacterial pathogens.

Several association studies have been conducted to find susceptibility related alleles on TLR2, TLR4, and TLR6 genes [34-37]. None of the previously identified susceptibility associated alleles or haplotypes were found in the present study in indigenous cattle breeds, however, all of them were identified in HOL.

Anatolian breeds seem to be more genetically resistant to infection with bacteria, such as Mycobacterium bovis and mastitis-causing bacteria and, when analyzing TLRs involved in the recognition of these bacterial pathogens, we indeed identified breed specific SNPs within the genes for the receptors investigated which differ significantly from those present in HOL cows.

Interestingly, when grouping the identified SNPs into haplotypes, Anatolian breeds grouped into different haplotypes for TLR2, but not for the other TLRs. This is an important observation, as those bacterial diseases, to which Anatolian breeds have been described to be more resistant, are mainly caused by bacteria binding to TLR2 [38]. Analyzing the identified SNPs in TLR2 in more detail, one aa change was identified in LRR1-10, four between LRR11-LRR20, one in the TM, and four in the TIR domains, respectively, in native cattle breeds, whereas only one was determined in LRR5 in HOL. Considering that the ligand-binding region of TLR2 encompasses LRR9-12, the most important change causing amino acid characteristic changes (H326Q) was found in LRR11. In addition, the aa changes identified in the TIR domain (H665Q and E738Q) need to be further investigated, as these might impact on subsequent intracellular signaling events, similar as described recently for bovine TLR5 [39].

In previous studies, aa changes L227P, H305P, and H326Q in the bovine TLR2 gene have been described to be under positive selection [10]. In our study, the aa at position 227 was L, whereas the aa at position 326 was $Q$, and no change was determined for the aa at position 305. Furthermore, aa changes L227P, H326Q, N417S, and H665Q have been identified as being specific to Bos indicus cattle breeds [10], similar to T405M [10], which we also identified in our study. It is currently assumed that these variations may represent geographical differences, being driven by a different microbiological environment. It has also been suggested that the Bos indicus-specific aa changes H326Q and $\mathrm{R} 563 \mathrm{H}$ might also be found in cattle breeds that originated in a similar geographical and microbial environment [10].

Indeed, blood parasites have been described to cause substantial economic losses in terms of production [40]. Genetic variations in TLR2 of Bos indicus cattle breeds are assumed to impact on blood parasite infections [41]. When the EAR hybrid cattle population breed in Diyarbakir were screened for 
Theileria blood parasite, only 24 out of 100 samples taken from clinically healthy cows tested positive for Theileria [42]. Similarly, while clinically healthy 24 EAR hybrid cattle $(n=111)$ and 21 Brown Swiss cows $(n=177)$ from Erzurum tested positive for the presence of Babesia spp. [43]. Taken further into account that previous phylogenetic studies concluded that Anatolian cattle breeds were Bos indicus and Bos taurus hybrids [44,45], we believe that either similar selective pressure may exists in Anatolian cattle breeds due to the similar geographic/microbial environment compared to pure Bos indicus breeds, or that Bos indicus breeds may have been crossed in at an earlier time due to an increase in the resistance of the local breeds to various infectious diseases.

In comparison with previous studies [34,46,47], the highest number of genetic variation in the analyzed genes were found in Anatolian breeds, except for TLR4, for which we identified more variations in the HOL breed, compared to $\mathrm{AB}$ and TG breeds. In these studies, researchers sampled a minimum one individual of each breed that went under artificial selection and analyzed innate immunity-related genes, partially. It is a known fact that the selection pressure for the quantitative traits associated with productions has a negative effect on immunity traits $[48,49]$. The cited studies showed that cattle breeds were under strong selection pressure, which might lead to a decrease in the variation on the gene regions. Nevertheless, with natural selection animals which cannot resist diseases and environmental conditions cannot find a chance for reproduction; thus, they are eliminated from the population [50] leading to accumulation of resistance-related variations. In the present study Anatolian cattle breeds were indigenous breeds that evolved under natural selection over the years. It can be assumed that the determined polymorphism and haplotype have a potential positive effect on immunity traits. In this context, when taking into account Figures 4 and 5, EAR and SAR, which were raised closer to the center of domestication, were seen as most divergent from the other breeds, suggesting more potential for disease resistance. Determination of the lowest haplotype number in the HOL breed might be due to a low sampling size and/or inbreeding for years. However the TG breed has the same sample size with the HOL breed, and 12 haplotypes were determined for TLR2 (Figure 4).

In addition to TLR2, we also assessed the occurrence of SNPs in TLR4 and TLR6, known to form heterodimers with TLR2. In the TLR6 gene, synonymous SNPs were positively associated with the susceptibility for bovine tuberculosis [51]. In the analyzed individuals of the presented study there were no breed-specific differences for the four synonymous variations. In addition to this, an SNP array study associated protein tyrosine phosphatase receptor T (PTPRT) and myosin IIIB (MYO3B) with bovine tuberculosis resistance [51]. The highest variation was identified in TLR6, but non-synonymous variations were seen in the LRR1-LRR14 in native cattle, whereas only one variation was observed in HOL cattle. One variation found in LRR14 may impact resistance and subsequently might be under positive selection as it was only detected in native cattle breeds [47].

With regards to TLR4, we identified two non-synonymous SNPs in LRR7 (N238K and A347E), which does not contribute to the MD2 binding region. The remaining variants identified all represented synonymous variations. The TLR4 region was analyzed for somatic cell score (SCS) and three SNPs were associated with high and low SCS values (intron1 rs8193046 A/G, exon3 rs8193060 C/T, and 5'UTR rs29017188 C/G) [52]. The ACC haplotype was associated with low SCS, whereas the GTG haplotype was associated with high SCS. High allele frequencies were found for the ACC haplotype in the TG cattle breed. TLR4 is also located in one of the QTL loci for milk production and mastitis [5]. Within the analyzed breeds, the AB breed has the lowest milk production and variation for TLR4, whereas highest variation number was determined in the high milk yield breeds, SAR, EAR and HOL as shown in Figure 5.

\section{Conclusions}

It is worth mentioning that the number of identified novel InDel variants is significantly higher compared to the identified number of SNPs. We believe that this can be attributed to the fact that InDels were mainly identified in intronic regions, whereas exonic regions in TLRs are more conserved, 
as well as the fact that the genetic composition Anatolian breeds, representing a hybrid of Bos taurus and Bos indicus breeds, has not been studied before. Given global warming due to climate change and increased anti-microbial resistance due to the overuse of antibiotics, new approaches are needed to manage infectious diseases in farm animals. A key step towards these is the identification of genotypes conferring resistance to both disease and adverse environmental conditions. Our identification of non-synonymous SNPs and novel variants of TLR2, TLR4, and TLR6 genes can provide one such set of variants for future association studies and the validation of the candidate haplotypes at a cellular level.

Supplementary Materials: The following are available online at www.mdpi.com/1424-2818/8/4/23/s1, Table S1: Oligonucleotide sequences were used in amplification; Table S2: PCR conditions and chemicals; Table S3: Determined haplotypes in TLR2, TLR4 and TLR6 gene regions.

Acknowledgments: This research has been supported by Ankara University Scientific Research Projects Coordination Unit. Project Number: 13B3338011, 2013; 14H0239004, 2014 and The Scientific and Technological Research Council of Turkey (TUBITAK): 1059B141400991, 2015. Authors would like to thank Martin Krzywinski for assistance in preparation of Figures 1-5.

Author Contributions: N.B.; B.C.K. and O.E. conceived and designed the experiments; N.B. and B.C.K. performed the experiments; N.B.; V.O.; and D.W. analyzed and interpret the data; N.B. and D.W. wrote the paper.

Conflicts of Interest: The authors declare no conflict of interest.

\section{References}

1. Kumar, H.; Kawai, T.; Akira, S. Pathogen recognition by the innate immune system. Int. Rev. Immunol. 2011, 30, 16-34. [CrossRef] [PubMed]

2. Medzhitov, R.; Janeway, C.A. Innate immunity: The virtues of a nonclonal system of recognition. Cell 1997, 91, 295-298. [CrossRef]

3. Tizard, I.R. Veterinary Immunology, 3rd ed.; Saunders Elsevier: Saint Louis, MO, USA, 2009; pp. 13-28.

4. Takeda, K.; Akira, S. TLR signaling pathways. Semin. Immunol. 2004, 16, 3-9. [CrossRef] [PubMed]

5. Ogorevc, J.; Kunej, T.; Razpet, A.; Dovc, P. Database of cattle candidate genes and genetic markers for milk production and mastitis. Anim. Genet. 2009, 40, 832-851. [CrossRef] [PubMed]

6. Huang, T.H.; Uthe, J.J.; Bearson, S.M.; Demirkale, C.Y.; Nettleton, D.; Knetter, S.; Tuggle, C.K. Distinct peripheral blood RNA responses to Salmonella in pigs differing in Salmonella shedding levels: İntersection of IFNG, TLR and miRNA pathways. PLoS ONE. 2011, 6, e28768. [CrossRef] [PubMed]

7. McGuire, K.; Jones, M.; Werling, D.; Williams, J.L.; Glass, E.J.; Jann, O. Radiation hybrid mapping of all 10 characterized bovine Toll-like receptors. Anim. Genet. 2006, 37, 47-50. [CrossRef] [PubMed]

8. Kent, W.J.; Sugnet, C.W.; Furey, T.S.; Roskin, K.M.; Pringle, T.H.; Zahler, A.M.; Haussler, D. The human genome browser at UCSC. Genome Res. 2002, 12, 996-1006. [CrossRef] [PubMed]

9. Cunningham, F.; Amode, M.R.; Barrell, D.; Beal, K.; Billis, K.; Brent, S.; Gil, L. Ensembl 2015. Nucleic Acids Res. 2015, 43, D662-D669. [CrossRef] [PubMed]

10. Jann, O.C.; Werling, D.; Chang, J.S.; Haig, D.; Glass, E.J. Molecular evolution of bovine Toll-like receptor 2 suggests substitutions of functional relevance. BMC Evol. Biol. 2008, 8, 288. [CrossRef] [PubMed]

11. Werling, D.; Jann, O.C.; Offord, V.; Glass, E.J.; Coffey, T.J. Variation matters: TLR structure and species-specific pathogen recognition. Trends Immunol. 2009, 30, 124-130. [CrossRef] [PubMed]

12. Schröder, N.W.; Schumann, R.R. Single nucleotide polymorphisms of Toll-like receptors and susceptibility to infectious disease. Lancet Infect. Dis. 2005, 5, 156-164. [CrossRef]

13. Alpan, O.; Arpacik, R. Sigir Yetiştiriciliği, 2nd ed.; Sahin Matbaası: Ankara, Turkey, 1996; pp. $39-46$.

14. Yilmaz, O.; Akin, O.; Yener, S.M.; Ertugrul, M.; Wilson, R.T. The domestic livestock resources of Turkey: Cattle local breeds and types and their conservation status. Anim. Genet. Resour. 2012, 50, 65-73. [CrossRef]

15. Özbeyaz, C. Sigir Yetiştiriciliği Ders Notlari; Ankara University, Faculty of Veterinary Medicine: Ankara, Turkey, 2015; pp. 10-26.

16. Rozen, S.; Skaletsky, H. Primer3 on the WWW for general users and for biologist programmers. Methods Mol. Biol. 1999, 132, 365-386.

17. Institue B. Picard. Available online: http://broadinstitute.github.io/picard (accessed on 11 May 2015).

18. Li, H.; Handsaker, B.; Wysoker, A.; Fennell, T.; Ruan, J.; Homer, N.; Durbin, R. The sequence alignment/map format and SAM tools. Bioinformatics 2009, 25, 2078-2079. [CrossRef] [PubMed] 
19. McKenna, A.; Hanna, M.; Banks, E.; Sivachenko, A.; Cibulskis, K.; Kernytsky, A.; DePristo, M.A. The Genome Analysis Toolkit: A MapReduce framework for analyzing next-generation DNA sequencing data. Genome Res. 2010, 20, 1297-1303. [CrossRef] [PubMed]

20. Danecek, P.; Auton, A.; Abecasis, G.; Albers, C.A.; Banks, E.; DePristo, M.A.; McVean, G. The variant call format and VCFtools. Bioinformatics 2011, 27, 2156-2158. [CrossRef] [PubMed]

21. Cingolani, P.; Patel, V.M.; Coon, M.; Nguyen, T.; Land, S.J.; Ruden, D.M.; Lu, X. Using Drosophila Melanogaster as a Model for Genotoxic Chemical Mutational Studies with a New Program, SnpSift. In Toxicogenomics in Non-Mammalian Species; Frontiers E-books: Lausanne, Switzerland, 2012; p. 35.

22. Cingolani, P.; Platts, A.; Wang, L.L.; Coon, M.; Nguyen, T.; Wang, L.; Ruden, D.M. A program for annotating and predicting the effects of single nucleotide polymorphisms, SnpEff: SNPs in the genome of Drosophila melanogaster strain w1118; iso-2; iso-3. Fly 2012, 6, 80-92. [CrossRef] [PubMed]

23. McLaren, W.; Pritchard, B.; Rios, D.; Chen, Y.; Flicek, P.; Cunningham, F. Deriving the consequences of genomic variants with the Ensembl API and SNP Effect Predictor. Bioinformatics 2010, 26, 2069-2070. [CrossRef] [PubMed]

24. Hall, T.A. BioEdit: A User-Friendly Biological Sequence Alignment Editor and Analysis Program for Windows 95/98/NT. Nucleic Acids Symp. Ser. 1999, 41, 95-98.

25. Webb, B.; Sali, A. Comparative Protein Structure Modeling Using Modeller. Curr. Protoc. Bioinform. 2014, 47, 1-32.

26. Laskowski, R.A.; MacArthur, M.W.; Moss, D.S.; Thornton, J.M. PROCHECK: A program to check the stereochemical quality of protein structures. J. Appl. Crystallogr. 1993, 26, 283-291. [CrossRef]

27. Eisenberg, D.; Lüthy, R.; Bowie, J.U. VERIFY3D: Assessment of protein models with three-dimensional profiles. Methods Enzymol. 1997, 277, 396-404. [PubMed]

28. Colovos, C.; Yeates, T.O. ERRAT: An empirical atom-based method for validating protein structures. Protein Sci. 1993, 2, 1511-1519. [CrossRef] [PubMed]

29. Wallner, B.; Elofsson, A. Can correct protein models be identified? Protein Sci. 2003, 12, 1073-1086. [CrossRef] [PubMed]

30. Thorvaldsdottir, H.; Robinson, J.T.; Mesirov, J.P. Integrative Genomics Viewer (IGV): High-performance genomics data visualization and exploration. Brief. Bioinform. 2013, 14, 178-192. [CrossRef] [PubMed]

31. Delaneau, O.; Howie, B.; Cox, A.; Zagury, J.F.; Marchini, J. Haplotype estimation using sequence reads. Am. J. Hum. Biol. 2013, 93, 787-696.

32. Bandelt, H.; Forster, P.; Rohl, A. Median joining networks for inferring intraspecific phylogenies. Mol. Biol. Evol. 1999, 16, 37-48. [CrossRef] [PubMed]

33. DeLano, W.L. PyMOL; DeLano Scientific: San Carlos, CA, USA, 2002; p. 700.

34. Mariotti, M.; Williams, J.L.; Dunner, S.; Valentini, A.; Pariset, L. Polymorphisms within the toll-like receptor (TLR)-2, -4, and-6 genes in cattle. Diversity 2009, 1, 7-18. [CrossRef]

35. Garza-Gonzalez, E.; Bosques-Padilla, F.J.; Mendoza-Ibarra, S.I.; Flores-Gutierrez, J.P.; Maldonado-Garza, H.J.; Perez-Perez, G.I. Assessment of the toll-like receptor 4 Asp299Gly, Thr399Ile and interleukin-8-251 polymorphisms in the risk for the development of distal gastric cancer. BMC Cancer 2007, 7, 70. [CrossRef] [PubMed]

36. Mucha, R.; Bhide, M.R.; Chakurkar, E.B.; Novak, M.; Mikula, I. Toll-like receptors TLR1, TLR2 and TLR4 gene mutations and natural resistance to Mycobacterium avium subsp paratuberculosis infection in cattle. Vet. Immunol. Immunopathol. 2009, 128, 381-388. [CrossRef] [PubMed]

37. Ruiz-Larrañaga, O.; Manzano, C.; Iriondo, M.; Garrido, J.M.; Molina, E.; Vazquez, P.; Estonba, A. Genetic variation of toll-like receptor genes and infection by Mycobacterium avium ssp. paratuberculosis in Holstein-Friesian cattle. J. Dairy Sci. 2011, 94, 3635-3641. [CrossRef] [PubMed]

38. Jungi, T.W.; Farhat, K.; Burgener, I.A.; Werling, D. Toll-like receptors in domestic animals. Cell Tissue Res. 2011, 343, 107-120. [CrossRef] [PubMed]

39. Osvaldova, A.; Woodman, S.; Patterson, N.; Offord, V.; Mwangi, D.; Gibson, A.J.; Matiasovic, J.; Werling, D. Replacement of two aminoacids in the bovine Toll-like receptor 5 TIR domain with their human counterparts partially restores functional response to flagellin. Dev. Comp. Immunol. 2014, 47, 90-94. [CrossRef] [PubMed]

40. Kaiser, P. The Immune System. In Breeding for Disease Resistance in Farm Animals, 3rd ed.; Bishop, S.C., Axford, R.F.E., Nicholas, F.W., Owen, J.B., Eds.; Cabi Publishing: Cambridge, MA, USA, 2010; pp. 15-38. 
41. Ropert, C.; Gazzinelli, R.T. Regulatory role of Toll-like receptor 2 during infection with Trypanosoma cruzi. J. Endotoxin Res. 2004, 10, 425-430. [CrossRef] [PubMed]

42. Deniz, A.; Oncel, T.; Icen, H.; Simsek, A. Detection of Theileria annulata and T. buffeli in Cattle by Multiplex PCR in Diyarbakir Area. Kafkas Univ. Vet. Fak. Derg. 2012, 18, A111-A114. [CrossRef]

43. Duzlu, O.; Yıldırım, A.; Inci, A.; Avcioglu, H.; Balkaya, I. Investigation of Babesia bovis and Babesia bigemina in cattle by Real Time PCR and molecular characterization of the isolates. Ankara Univ. Vet. Fak. Derg. 2015, 62, 27-35.

44. Loftus, R.T.; Ertuğrul, O.; Harba, A.H.; El-Barody, M.A.; Machugh, D.E.; Park, S.D.; Bradley, D.G. A microsatellite survey of cattle from a center of origin: The Near East. Mol. Ecol. 1999, 8, 2015-2022. [CrossRef] [PubMed]

45. Decker, J.E.; McKay, S.D.; Rolf, M.M.; Kim, J.; Alcalá, A.M.; Sonstegard, T.S.; Hanotte, O.; Götherström, A.; Seabury, C.M.; Praharani, L.; et al. Worldwide patterns of ancestry, divergence, and admixture in domesticated cattle. PLoS Genet. 2014, 10, e1004254. [CrossRef] [PubMed]

46. Seabury, C.M.; Seabury, P.M.; Decker, J.E.; Schnabel, R.D.; Taylor, J.F.; Womack, J.E. Diversity and evolution of 11 innate immune genes in Bos taurus taurus and Bos taurus indicus cattle. Proc. Natl. Acad. Sci. USA 2010, 107, 151-156. [CrossRef] [PubMed]

47. Fisher, C.A.; Bhattarai, E.K.; Osterstock, J.B.; Dowd, S.E.; Seabury, P.M.; Vikram, M.; Womack, J.E. Evolution of the bovine TLR gene family and member associations with Mycobacterium avium subspecies paratuberculosis infection. PLoS ONE 2011, 6, e27744. [CrossRef] [PubMed]

48. Ingvartsen, K.L.; Dewhurst, R.J.; Friggens, N.C. On the relationship between lactational performance and health: Is it yield or metabolic imbalance that cause production diseases in dairy cattle? A position paper. Livest. Prod. Sci. 2003, 83, 277-308. [CrossRef]

49. Oltenacu, P.A.; Broom, D.M. The impact of genetic selection for increased milk yield on the welfare of dairy cows. Anim. Welf. 2010, 19, 39-49.

50. Haldane, J.B.S. Disease and evolution. Curr. Sci. 1992, 63, 599-604.

51. Bermingham, M.L.; Bishop, S.C.; Woolliams, J.A.; Pong-Wong, R.; Allen, A.R.; McBride, S.H.; Glass, E.J. Genome-wide association study identifies novel loci associated with resistance to bovine tuberculosis. Heredity 2014, 112, 543-551. [CrossRef] [PubMed]

52. De Mesquita, A.Q.; Rezende, C.S.M.; de Mesquita, A.J.; Jardim, E.A.G.; Kipnis, A.P.J. Association of TLR4 polymorphisms with subclinical mastitis in Brazilian holsteins. Braz. J. Microbiol. 2012, 43, 692-697. [CrossRef] [PubMed]

(C) 2016 by the authors; licensee MDPI, Basel, Switzerland. This article is an open access article distributed under the terms and conditions of the Creative Commons Attribution (CC-BY) license (http://creativecommons.org/licenses/by/4.0/). 